\title{
Prediction of Local Losses of Low $R e$ Flows in Non-uniform Media Composed of Parrallelpiped Structures
}

\author{
Sid M. Becker ${ }^{1}$ (D)
}

Received: 28 September 2017 / Accepted: 4 January 2018

(C) Springer Science+Business Media B.V., part of Springer Nature 2018

\begin{abstract}
A method is presented to predict the local losses of low $R e$ flow through a porous matrix composed of layers of orthogonally oriented parallelepipeds for which the local geometry varies discreetly in the direction of bulk flow. In each layer, the variations in the pore lengths perpendicular to and parallel to the direction of bulk flow are restricted to be proportional to one another so that the variation in the geometry of each layer may be characterized by a single parameter, $\beta$. The solutions to the Navier-Stokes equations are determined for flows through geometries that vary in a forward expansion about this parameter. These provide the data used in the development of a correlation that is able to directly relate local hydraulic permeability to the variation in local pore geometry. In this way, the local pressure losses (as well as the relationship between the volumetric flow rate and the total pressure drop) may be determined without requiring the explicit solution of the entire flow field. Test cases are presented showing that the correlation predicts the local pressure losses to be within $0.5 \%$ of the losses determined from the numerical solution to the Navier-Stokes equations. When the magnitude of the variation to the geometry is such that the change in the parameter $\beta$ between layers is constant throughout the medium, a reduced form of the correlation (requiring the evaluation of only three constants) is able to provide predictions of flow rate and interface pressures that agree to within about $1 \%$ with the results of the numerical solutions to the Navier-Stokes equations.
\end{abstract}

Keywords Permeability prediction - Correlation · Darcy flow · Parallelpiped - Deterministic model

\section{List of symbols}

$\beta \quad$ Variation parameter

Sid M. Becker

sid.becker@canterbury.ac.nz

1 University of Canterbury, Christchurch, New Zealand 
$\Delta \beta^{+} \quad$ Downstream change in variation parameter

${ }_{24} \Delta \beta^{+}$

$25 \mu$

Upstream change in variation parameter

$\mu \quad$ Fluid viscosity

$v \quad$ Ratio of lateral variation to longitudinal variation

$A_{\mathrm{T}} \quad$ Total cross-sectional area (void plus solid)

$\ell \quad$ Lateral side length scale associated with the solid matrix

$L \quad$ Side length corresponding to layer height

$L_{0} \quad$ Unperturbed side length corresponding to layer height

$\Delta L \quad$ Difference between the unperturbed layer height and the perturbed layer height

$K \quad$ Permeability

$N \quad$ Number of layers

$\Delta P \quad$ Difference in average pressure

$\Delta P_{\mathrm{T}} \quad$ Difference in average total pressure

$Q \quad$ Volumetric flow rate (in the direction of bulk flow)

$U \quad$ Seepage velocity

\section{Subscript}

$i \quad$ Layer number

\section{Introduction}

It is well understood that at low $R e$, flows through uniform porous media exhibit a linear relationship between the pressure gradient in the direction of flow and the seepage velocity.

$$
\frac{\partial p}{\partial z}=-\frac{U \mu}{K}
$$

Here, $\mu$ is the dynamic viscosity and $K$ is the permeability of the porous media (with dimensions of length squared). The seepage velocity is related to the volumetric flow rate, $Q$, by:

$$
U=\frac{Q}{A_{\mathrm{T}}}
$$

were $A_{\mathrm{T}}$ is the total cross-sectional area (void plus solid) normal to the direction of bulk flow. The permeability may be determined experimentally or numerically from the relation:

$$
K=U \mu\left(\frac{\Delta P}{\Delta L}\right)^{-1}
$$

Here, $\Delta L$ is the length of the porous medium over which the fluid experiences a difference in average pressure, $\Delta P$. The permeability of the porous medium is determined only from studies or simulations of flows that have no significant inertial contributions. Consider Fig. 1 which depicts the right-hand side of Eq. (3) evaluated from the results of simulations at different Reynolds numbers. As anticipated, at low $R e$, the permeability is independent of $R e$ and is only dependent on the pore geometry (here the magnitude of the symbol $\beta$ is used to indicate a difference in matrix geometry and this symbol is defined later).

The permeability is always a function of geometry regardless of any heterogeneity in the flow field (Mathieu-Potvin and Gosselin 2014), and the prediction of the permeability (given full knowledge of matrix geometry) is of great interest to researchers. Of particular interest 


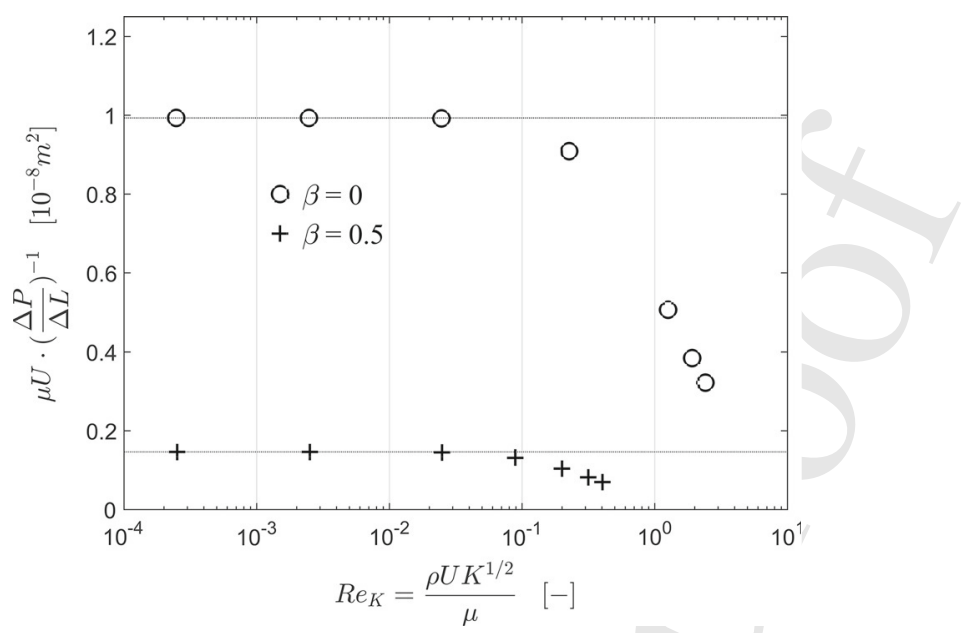

Fig. 1 An illustration of the relationship between the permeability and the Reynolds number for media characterized by two different geometries $(\beta=0$ and $\beta=0.5)$

to the current study is the determination of the permeability of a matrix that has characteristic lengths that vary within the medium. The importance of considering the local pore structure for media with geometry that varies in the direction perpendicular to the direction of bulk flow has been well studied, see for example the seminal work by Vafai (1984). The descriptions of slurry flow through evolving dendritic structures in Goyeau et al. (1997, 1999) emphasize that macroscopic modeling is greatly improved when local heterogeneity in the structure is taken into account.

The current study is interested in the prediction of the permeability when the geometry varies in the direction of bulk flow. A comprehensive introduction and review of the field's body of knowledge regarding the relationship between matrix geometry and permeability is presented in book by Dullien (1992). That book distinguishes itself by using a frame of reference that is focused on the pore structure first and then the fluid mechanics after. One of the pore structures presented therein is represented by bundles of capillary tubes that experience periodic constrictions and expansions. Permeability predictions that consider such serial type changes in tube geometry have been presented that relate the permeability to the pore diameters, porosity, and a pore size density (Dullien and Azzam 1973, 1976; Azzam and Dullien 1977; Dullien et al. 1977).

More ordered matrix geometries are sometimes represented by pore networks that include parallelepiped geometries (Barrer and Petropoulos 1961; Mazaheri et al. 2005). Flows through similar geometries have also been studied in fuel cell applications (Tehlar et al. 2010). The geometry of the current study is motivated by the physical media developed and fabricated in the experimental work (Munro et al. 2015).

The current study considers flow through a porous structure that is composed of regularly distributed orthogonally oriented parallelepipeds. Consider the regular periodic geometry representative of the Cartesian matrix structure depicted in Fig. 2. This matrix is composed of distinct layers of uniformly distributed parallelepipeds and these layers are "stacked" in the direction of bulk flow (along the $z$ coordinate direction). The longitudinal axis of each parallelepiped is perpendicular to the direction of bulk flow. For each layer, this axis is also oriented perpendicular to those of the adjacent layers. The parallelepipeds of alternating 

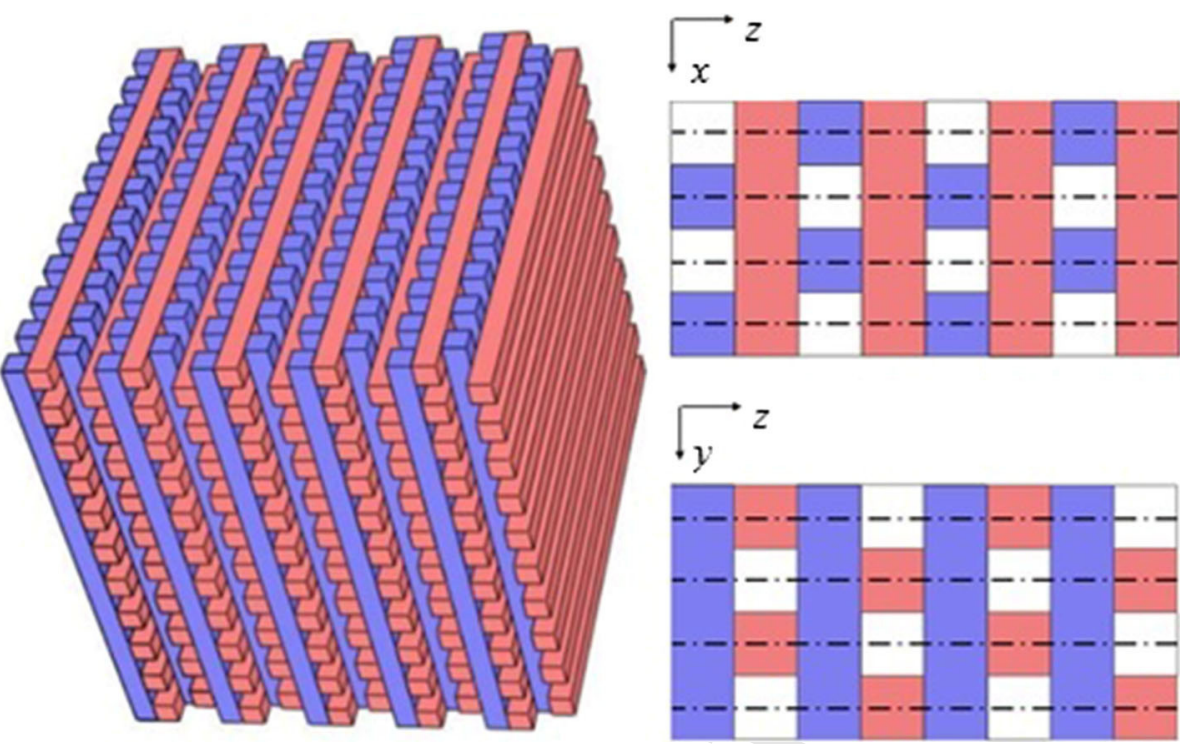

Fig. 2 Porous structure depicted in 3-d (left); Side views in the $x-z$ and $y-z$ planes with dash-dot lines indicating planes of symmetry (right). Note that the planes of symmetry bisect the pores in lateral directions

parallel layers are offset by a distance of one half a pore thickness; this introduces tortuosity to the path of the fluid. Symmetry exists in the matrix structure and it is anticipated that similar planes of symmetry exist in the flow (indicated by the dash-dot lines in Fig. 2). Between these planes of symmetry the flow paths through the medium may be individually represented by a single representative pore structure. This is illustrated in Fig. 3a that depicts the fluid filled space of an individual pathway through 6 layers of the uniform medium. The periodic nature of this flow structure is immediately evident and, for a uniform medium, the rectangular parallelepiped structure of each layer's void space (Fig. 3b) is an identical copy of that of the preceding layer but with a $270^{\circ}$ rotation about the $z$ axis.

Because this study is interested in the case in which the geometrical scales characterizing the matrix vary in the direction of flow, it is helpful to characterize the geometry of each layer in terms of a variation about the unperturbed layer height, $L_{0}$. In this work, a variation parameter, $\beta$, is introduced in order to characterize the deviation from the unperturbed layer height (which is also the pore length in the direction of bulk flow). The variation parameter of the $i$ th layer (which is analogous to the strain in the solid matrix of that layer undergoing a perfectly elastic deformation) is defined as the ratio of the variation in the height of the $i$ th layer, $\Delta L_{i}$, to the unperturbed layer height, $L_{0}$ :

$$
\beta_{i} \equiv \frac{\Delta L_{i}}{L_{0}}
$$

Here, the variation in the height of the $i$ th layer is the difference between the unperturbed layer height and the perturbed layer height, $\Delta L_{i}=L_{0}-L_{i}$. In this way, the pore length in the direction of bulk flow $(z)$ of the $i$ th layer of the medium is linearly dependent on the variation parameter by the relation:

$$
L_{i}=L_{0}\left(1-\beta_{i}\right)
$$




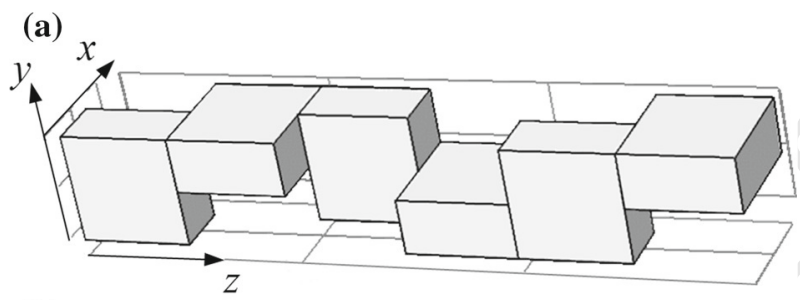

(b)

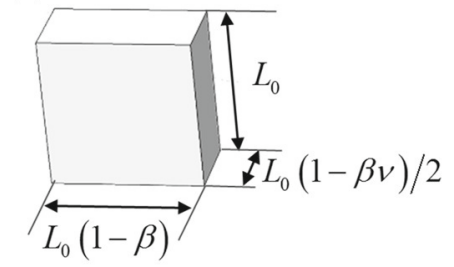

(c)

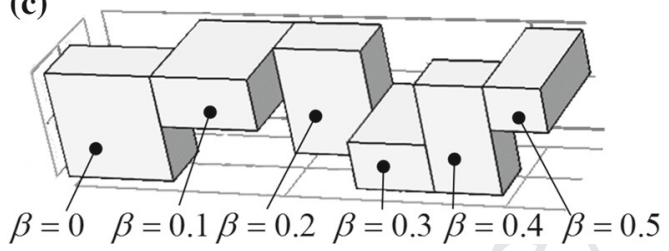

Fig. 3 a The representative pore structure through the uniform Cartesian matrix (constant $\beta$ ), b the geometric characterization of the pore structure in a single layer, $\mathbf{c}$ the representative pore structure for a non-uniform medium in which each layer's variation parameter, $\beta$, increases in the direction of flow

Consider next the lengths of the pore sides that are perpendicular to the direction of flow (oriented, respectively, along the $x$ and $y$ coordinates). The length of one of these sides is always equal to the constant $L_{0}$, while the length of the other side may vary between layers and the orientations of these side lengths alternate coordinate directions ( $x$ or $y)$ between adjacent layers. The length of the side that is free to experience a contraction or an expansion is linearly related to the variation parameter by some positive constant, $v$. In this way, the $i$ th layer's pore length perpendicular to the direction of bulk flow may be described by the relation:

$$
\ell_{i}=\frac{L_{0}}{2}\left(1-\varepsilon_{i} v\right)
$$

The parameter $v$ is analogous to the Poisson ratio of a material undergoing elastic compression or elongation. The porosity of any layer of the porous medium is related to the variation parameter by the expression:

$$
\varphi_{i}=\left(1-\beta_{i} v\right) / 2
$$

The parameter $v$ is treated as a constant, however the magnitude of the variation parameter, $\beta$, is free to change between layers. An example of a non-uniform structure for which the variation parameter changes between layers is depicted in Fig. 3c. It is the intent of this study to develop a method to predict the relationship between pressure drop and flow rate for the case in which the porous media experiences a discreet (layer by layer) change in local pore geometry.

Take into account the well-structured phrase from page 6 of the excellent book (Nield and Bejan 2013): 
"If $K$ is indeed determined by the geometry of the medium, then clearly it is possible to calculate $K$ in terms of the geometrical parameters, at least for the case of simple geometry."

In this paper, this statement is examined in the context of a non-uniform porous medium for which the variation in that geometry may be characterized by the single parameter, $\beta$. When the value of $\beta$ in the medium varies discreetly between layers of the medium, and when its value in each layer is known, it seems plausible that the permeability of any layer " $i$ " may be described to have a dependency only on the values of the variation parameter (i) of that layer $\beta_{i}$, (ii) of its upstream neighbor $\beta_{i-1}$, and (iii) of its downstream neighbor $\beta_{i+1}$. This study proposes to determine the functional relationships between the dimensionless parameters:

$$
\frac{K_{i}}{L_{0}^{2}}=f\left(\beta_{i-1}, \beta_{i}, \beta_{i+1}\right)
$$

The results of numerical simulations are used to investigate the nature of this dependence, and then these results are used to determine a best fit curve to predict the dependence of permeability on the local variation parameters.

\section{Numerical Simulations and Computational Considerations}

While numerical simulations are not the focus of this study, this paper will develop correlations using the results of numerical simulations, and thus this section provides some details of the numerical computations here. These simulations are conducted using the Software package Comsol version 4.3a. An Intel@ Core ${ }^{\mathrm{TM}}$ i7-4790 CPU @ $3.60 \mathrm{GHz}$ with $32.0 \mathrm{~GB}$ RAM is used to run the simulations. The stationary "Laminar Flow" model of the "Single Phase Flow" model library is used to determine the solutions to the continuity and NavierStokes momentum equations with constant fluid properties. Symmetry boundary conditions are implemented on all surfaces corresponding to planes of symmetry (these correspond to the outer $x-z$ and $y-z$ planes of Fig. 3a-c), and the no-slip boundary condition is imposed on surfaces corresponding to the fluid-solid interfaces. The "Finer" setting of the "Physics-controlled mesh" is used following grid refinement studies that were conducted until increased refinement resulted in changes of less than $0.05 \%$ in the resulting flow rate and the average pressures at specified planes.

At the inlet and outlet of the pore structure, uniform pressures are specified. To ensure that the permeability calculated is $R e$ independent, for each simulation, the total pressure drop was chosen $\left(\Delta P_{\mathrm{T}} \leq 100 \mathrm{~Pa}\right)$ so that in all simulations the local Reynolds number remains below $1.7 \times 10^{-3}$. In this study, the local Reynolds number is defined using the seepage velocity of Eq. (2) to represent the characteristic velocity and the characteristic length is represented by the square root of the permeability (see Fig. 1). Simulations considered the flow of a viscous liquid with a density of $10^{3} \mathrm{~kg} \mathrm{~m}^{-3}$ and a viscosity of $0.1 \mathrm{~Pa} \mathrm{~s}^{-1}$.

The numerical results of the flow field were post-processed in Comsol and MatLab. The Comsol numerical integrator, "Surface Integration", is used to determine the volume flow rate at specified cross sections perpendicular to the direction of bulk flow. The "Surface Average" tool is used to determine the average pressure along the planes in the fluid domain that correspond to the interfaces between the layers. For each geometric configuration, the flow rate and average pressures are exported and then in a MatLab script the permeability of each simulation is calculated. The MatLab function "Isqcurvefit" is used to determine the least squares best fit curve for the correlations which are described next. 


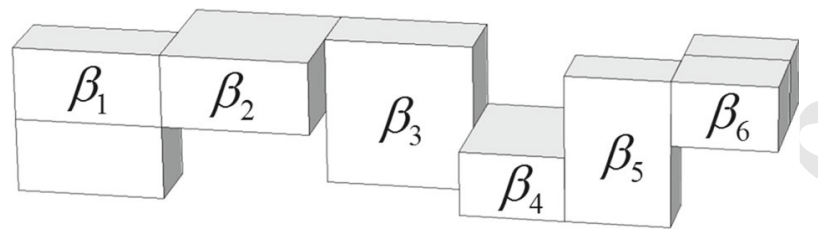

Fig. 4 The representative geometry of the pore structure used in the simulations to determine in the permeability data used in the correlations

\section{Correlations}

It is anticipated that the dimensionless representation of the local permeability of low $R e$ flows through non-uniform porous media is dependent only on the local pore geometry. As implied by Eq. (8), with respect to the geometry described in Fig. 3b, the dimensionless permeability of any particular layer is proposed to depend only on the variation parameter of that layer and on the variation parameters associated with the adjacent layers. This section describes the manner in which a correlation is developed using the data of flow.

The pore geometries upon which the simulations are conducted consist of variations on the 6 layer pore structure depicted in Fig. 4. The first three layers always have the same variation parameter $\beta_{1}=\beta_{2}=\beta_{3}$ and the last three layers always have the same variation parameter $\beta_{4}=\beta_{5}=\beta_{6}$ so that it is possible to introduce an abrupt and discrete change in the variation parameter at the interface between layer 3 and layer 4 . The side lengths of each layer's pore structure are determined by relations (5) and (6) with an unperturbed side length of $L_{0}=10^{-3} \mathrm{~m}$. In all cases presented in this study, the parameter, $v$, that appears in Eq. (6) and relates the variation in the lateral side length to the variation in layer height is constant and equal to $v=0.4$.

When the geometry is uniform, there is no abrupt change in variation parameter $\left(\beta_{3}=\beta_{4}\right)$ and the permeability is anticipated to be uniform throughout the medium. Alternately for any abrupt change in variation parameter $\left(\beta_{3} \neq \beta_{4}\right)$, it is anticipated that there will be a corresponding change in medium permeability. To simplify the notation used in the following analysis, a downstream change in the variation parameter of layer " $i$ " is defined as:

$$
\Delta \beta_{i}^{-} \equiv \beta_{i-1}-\beta_{i}
$$

and an upstream change in the variation parameter of layer " $i$ " is defined as:

$$
\Delta \beta_{i}^{+} \equiv \beta_{i+1}-\beta_{i}
$$

Following each simulation, the surface averaged fluid pressure is calculated over the three planes perpendicular to the direction of bulk flow: one at the interface between layer 2 and layer 3, one at the interface between layer 3 and layer 4 , and one at the interface between layer 4 and layer 5 . For each layer $(i=3$ and $i=4)$, the difference in the surface averaged pressure, $\Delta P_{i}$, is determined and each of these occurs over the layer height $L_{i}$ which can be related to the local variation parameter though Eq. (5)

For each simulation, the volumetric flow rate in the direction of bulk flow, $Q$, is also calculated. The total cross-sectional area in the direction of bulk flow for this configuration is $A_{\mathrm{T}}=L_{0}^{2}$ so that the seepage velocity of Eq. (2) may be related to the volumetric flow rate, $Q$, by:

$$
U=\frac{Q}{L_{0}^{2}}
$$


From the data of each simulation, two values of the local permeability (one for layer $i=3$ and one for layer $i=4$ ) are calculated as:

$$
K_{S, i}=\frac{U \mu}{\left(\Delta P_{i} / L_{i}\right)}
$$

In this way, it is possible to relate each deterministically evaluated permeability value, $K_{S}$, to its corresponding values of $\beta, \Delta \beta^{-}$, and $\Delta \beta^{+}$. A brief discussion on the domain independence of the calculated local permeability values of layer 3 and layer 4 is presented in the appendix of this study.

In order to provide insight into the general dependence of local permeability on $\beta, \Delta \beta^{+}$, and $\Delta \beta^{-}$, a preliminary investigation is conducted. Any increase in the variation parameter will result in a decrease in pore dimensions (and thereby the porosity) so it makes sense that permeability will decrease with increasing $\beta$. This is confirmed in Fig. 5a that depicts the dependence of the permeability on the variation parameter in a uniform media $\left(\Delta \beta^{+}=\right.$ $\Delta \beta^{-}=0$ ). This dependence appears quadratic at lower values of $\beta$. In the range $0 \leq \beta \leq 0.5$, a best fit quadratic curve (represented by the dash-dot line in Fig. 5a) results in an excellent representation of the data. As the variation parameter increases, this dependence smoothly transitions into what appears to be a negative exponential dependence which is depicted in the insert panel of Fig. 5a.

Note that increasing the values of either $\Delta \beta^{+}$or $\Delta \beta^{-}$always results in a decrease in the cross-sectional area of the fluid path at the interface between the layers. This will result in an increase in the resistance to the flow thereby lowering the permeability. Figure $5 \mathrm{~b}$ depicts the influence that the change in variation parameter has on the permeability (although only the influence of downstream change in the variation parameter is presented here). Here, the values of permeability calculated from the simulation data are plotted for 4 different values of the variation parameter over a range of $\Delta \beta^{+}$. For each variation parameter, the permeability has a highly linear dependence on $\Delta \beta^{+}$, and the slope of this dependence varies with $\beta$. It appears that within the range $-0.2 \leq \Delta \beta^{ \pm} \leq 0.2$, for each $\beta$, the permeability may safely be expressed using a linear dependence on $\Delta \beta^{ \pm}$.

The results of the preliminary investigation imply that the local permeability may be represented with a quadratic dependence on $\beta$ in a uniform medium. In a non-uniform medium, the local permeability may be expressed with a linear dependence on $\Delta \beta^{ \pm}$and the slope of this linear dependence is also influenced by $\beta$. For porous geometries in which the variation in geometry is constrained such that $0 \leq \beta \leq 0.5$ and $-0.2 \leq \Delta \beta^{ \pm} \leq 0.2$, the following dimensionless relationship is proposed:

$$
\frac{K}{L_{0}^{2}}=a_{1}+a_{2} \beta+a_{3} \beta^{2}+\Delta \beta^{-}\left(a_{4}+a_{5} \beta\right)+\Delta \beta^{+}\left(a_{6}+a_{7} \beta\right)
$$

The non-inertial losses associated with the flow through each layer of the pore structure result from the three contributions: (i) viscous resistance associated with the flow through each layer, (ii) viscous losses resulting from the sudden expansion at each layer's inlet, and (iii) viscous losses resulting from the sudden contraction at each layer's outlet. When the medium is uniform, these three contributors are represented by the first three terms of Eq. (13). Non-uniformities in the porous medium result in changes to the cross-sectional area at the interface between layers of different $\beta$. These, in turn, will influence the losses associated with the expansion and contraction of the flow at that interface. The deviation from the permeability associated with a uniform medium is represented by the remaining terms of Eq. (13). 
Fig. 5 Preliminary observations of the behavior of the permeability evaluated directly from the numerical solutions to the Navier-Stokes equations. a uniform matrix geometries in which $\Delta \beta^{+}=\Delta \beta^{-}=0$; the dash-dot line is the best fit quadratic curve to the data in the range $0 \leq \beta \leq 0.5$, b variable matrix geometries that experience a change in $\Delta \beta^{+}$between layers; the dash-dot lines are the best fit linear curves of the data in this region
Table 1 Permeability values calculated from Eq. (12) using the results of simulations of flows through seven different variations of the geometry presented in Fig. 1
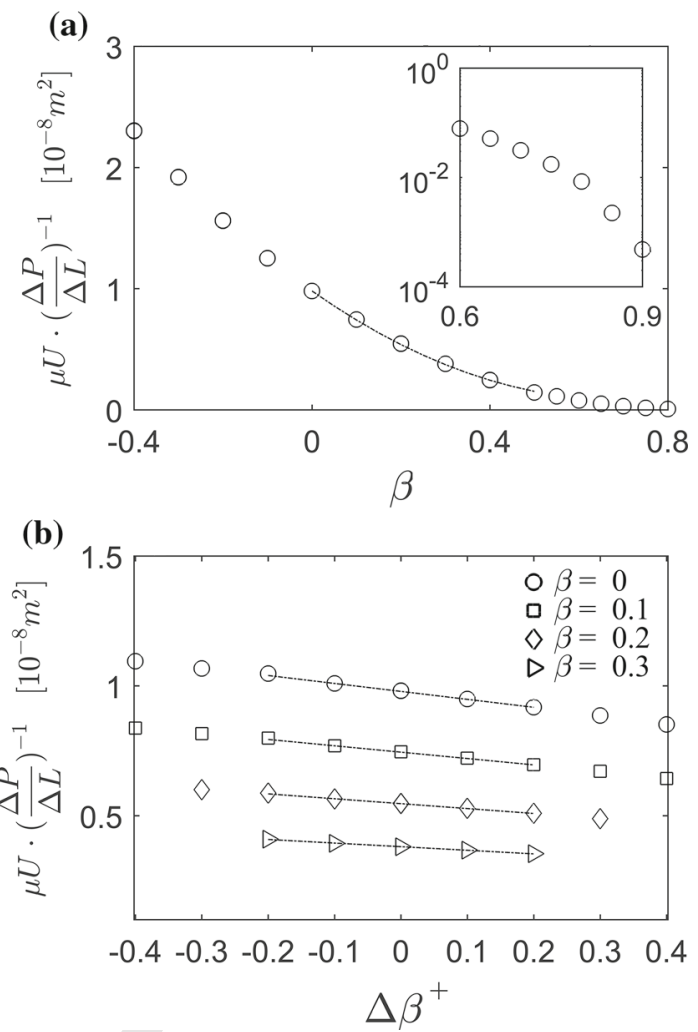

\begin{tabular}{llll}
\hline$\beta$ & $\Delta \beta^{+}$ & $\Delta \beta^{-}$ & $K_{S} / L_{0}^{2}$ \\
\hline 0.3 & 0 & 0 & $3.813 \mathrm{E}-03$ \\
0 & 0 & 0 & $9.821 \mathrm{E}-03$ \\
0.5 & 0 & 0 & $1.446 \mathrm{E}-03$ \\
0.3 & 0.2 & 0 & $3.541 \mathrm{E}-03$ \\
0.5 & 0 & -0.2 & $1.549 \mathrm{E}-03$ \\
0.2 & -0.2 & 0 & $5.878 \mathrm{E}-03$ \\
0 & 0 & 0.2 & $9.256 \mathrm{E}-03$ \\
0.4 & 0.1 & 0 & $2.386 \mathrm{E}-03$ \\
0.5 & 0 & -0.1 & $1.499 \mathrm{E}-03$ \\
0.1 & -0.1 & 0 & $7.700 \mathrm{E}-03$ \\
0 & 0 & 0.1 & $9.498 \mathrm{E}-03$
\end{tabular}

In order to evaluate the constants of this correlation, seven unique variations of the geometry described by Fig. 4 are considered and simulated. The combinations of $\beta, \Delta \beta^{-}$, and $\Delta \beta^{+}$along with the corresponding permeability magnitudes resulting from the numerical simulations Eq. (12) are listed in Table 1.

The constants $a_{1}-a_{7}$ of Eq. (13) are then determined using the method of least squares from this data. 
(a) Case 1: Constant $\Delta \beta^{ \pm}$

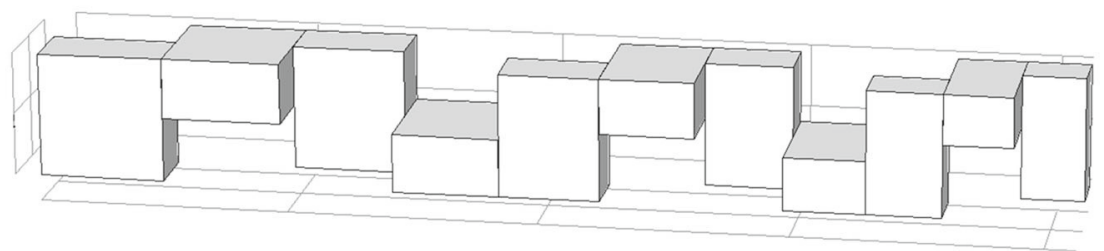

(b) Case 2: Variable $\Delta \beta^{ \pm}$

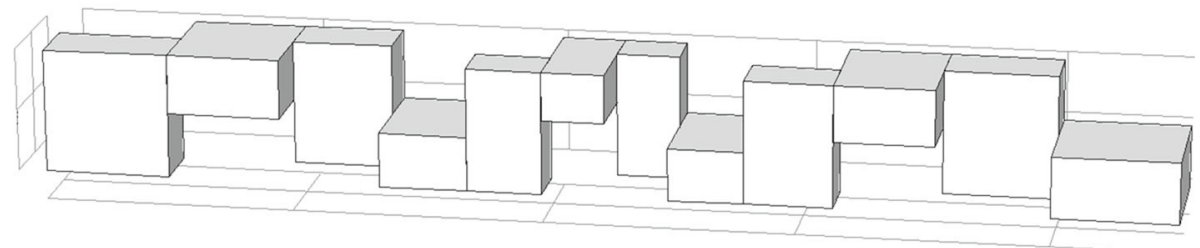

Fig. 6 A depiction of the geometry of the two test cases: a an 11 layer structure with a uniform change in variation parameter for which the variation parameters may be represented by the array $[00.05,0.1,0.150 .2$, $0.25,0.3,0.35,0.4,0.45,0.5] ; \mathbf{b}$ a 12 layer structure with a non-uniform change in variation parameter for

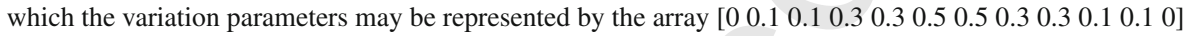

These are listed in Eq. (14)

$$
\begin{array}{ll}
a_{1}=9.802 \mathrm{E}-03 & a_{2}=-2.478 \mathrm{E}-02 \\
a_{4}=-2.774 \mathrm{E}-03 & a_{5}=4.556 \mathrm{E}-03 \\
a_{6}=-2.790 \mathrm{E}-03 & a_{7}=4.586 \mathrm{E}-03
\end{array}
$$

As a measure of the accuracy, a comparison is conducted between the predicted values of the correlation (13) with the constants (14) and the values of the permeability determined from Eq. (12) using data determined from many more simulations. Sixty two points of comparison are considered resulting from 34 different simulations of flow through variations of the geometry of Fig. 4 with all combinations of $\left|\Delta \beta_{3}^{+}\right|=[0,0.05,0.1,0.2]$ and $\beta_{3}=[0,0.1,0.2,0.3,0.4,0.5]$ that fall into the specified ranges $0 \leq \beta \leq 0.5$ and $\left|\Delta \beta^{ \pm}\right| \leq 0.2$. The root-mean-square difference of the 62 permeability values determined from the simulations and from the correlation was rmsd $=2.539 \mathrm{E}-5$ and the coefficient of determination was $r^{2}=0.9999$.

\section{Test Cases}

In this section, the predictions of flows through the two different geometries depicted in Fig. 6 are presented. In these test cases, the variation parameter of each layer, $\beta_{i}$, is specified and the total pressure drop $\Delta P_{\mathrm{T}}$ over the porous medium is specified. For each test case, the predicted permeability of each layer of the structure is first evaluated from constants of Eq. (14) with the correlation:

$$
\frac{K_{i}}{L_{0}^{2}}=a_{1}+a_{2} \beta_{i}+a_{3} \beta_{i}^{2}+\Delta \beta_{i}^{+}\left(a_{4}+a_{5} \beta_{i}\right)+\Delta \beta_{i}^{-}\left(a_{6}+a_{7} \beta_{i}\right) \quad i=1,2 \ldots, N
$$

\section{Springer}


Fig. 7 Uniform change in variation parameter

$\Delta \beta_{i}^{+}=-\Delta \beta_{i+1}^{-}=0.05$. The comparison between Numerical Simulation and the full correlation of Eq. (15) of a the difference in average pressure across each layer, and $\mathbf{b}$ the pressure at each layer outlet

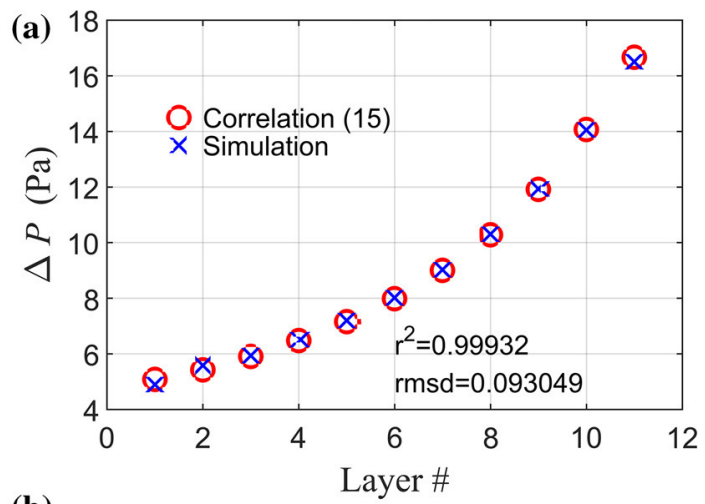

(b)

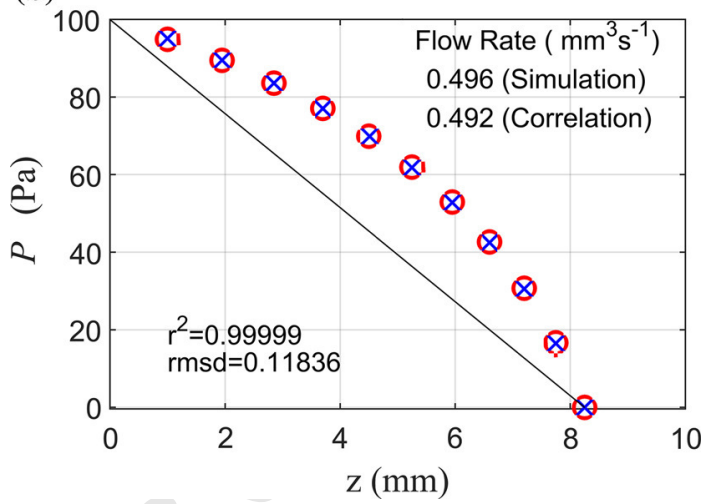

Here, $N$ is the total number of layers. Note that the upstream change in the variation parameter of the first layer and the downstream change in the variation parameter of the last layer are in this study neglected by the approximations $\Delta \beta_{1}^{-}=\Delta \beta_{N}^{+}=0$. The one-dimensional steady representation of Darcy flow through the serial type layered geometry is rewritten using a resistor representation so that the volumetric flow rate is related to the total pressure drop by the relation:

$$
Q=\frac{\Delta P_{\mathrm{T}} A_{\mathrm{T}}}{\mu} \frac{1}{\sum_{i=1}^{N}\left(L_{i} / K_{i}\right)}
$$

where each layer height, $L_{i}$, is related to its variation parameter by Eq. (5). The prediction of the drop in the average pressures across each layer may then be evaluated from the relation:

$$
\Delta P_{i}=\frac{\mu}{\left(K_{i} / L_{i}\right)} \frac{Q}{L_{0}^{2}} \quad i=1,2, \ldots, 12
$$

Alternately, if the volumetric flow rate were specified (instead of the total pressure drop), Eq. (17) could be immediately used to evaluate the local pressure drop and Eq. (16) could be rearranged in order to determine the total pressure drop.

In the following test cases, the inlet and outlet pressures (gage) are specified to be $100 \mathrm{~Pa}$ and $0 \mathrm{~Pa}$, respectively. The fluid density is $10^{3} \mathrm{~kg} \mathrm{~m}^{-3}$ and the fluid viscosity is $0.1 \mathrm{~Pa} \mathrm{~s}^{-1}$. The geometric parameters used are $L_{0}=10^{-3} \mathrm{~m}$ and $v=0.4$.

The first test case, depicted in Fig. 6a, is an 11 layer structure that has a uniformly increasing value of the variation parameter so that the first layer has a variation parameter of $\beta_{1}=0$ and 
Fig. 8 Uniform change in variation parameter $\Delta \beta_{i}^{+}=-\Delta \beta_{i+1}^{-}=0.05$. The comparison between Numerical Simulation and the Reduced Correlation of Eq. (18) of a the difference in average pressure across each layer, and $\mathbf{b}$ the pressure at each layer outlet

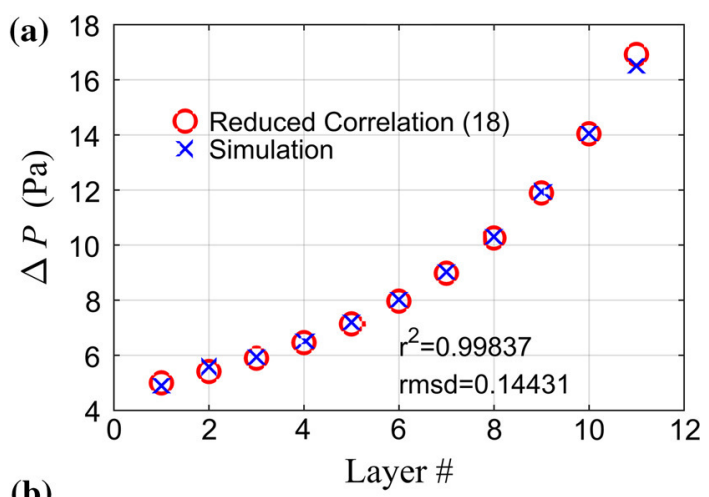

(b)

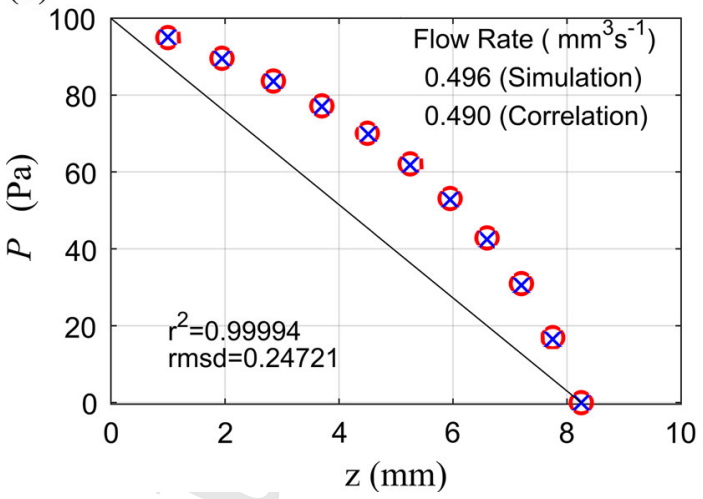

each subsequent layer's variation parameter increases by $0.05\left(\Delta \beta_{i}^{+}=0.05 i=1, \ldots, 10\right.$ and $\left.\Delta \beta_{i}^{-}=-0.05 i=2, \ldots, 11\right)$. In this way, the variation parameters are represented by the array $[00.05,0.1,0.150 .2,0.25,0.3,0.35,0.4,0.45,0.5]$. A comparison between the results of the numerical simulations and the predictions of the correlation resulting from Eqs. (13-17) is presented in Fig. 7. The pressure drop over each layer (Fig. 7a) increases with increasing $\beta$ in a quadratic manner (as is anticipated), and the correlation's predictions agree well with the simulation. The calculated average pressure at each layer's outlet is depicted in Fig. 7b also showing excellent agreement. A solid line has been added here to accentuate the deviation of this pressure distribution from that represented by a flow exhibiting a uniform pressure gradient (the magnitude of the slope of this line is proportional to the effective permeability of the medium). The correlation's predicted volumetric flow rate of Eq. (16) agrees to within $1 \%$ of that determined from the results of the numerical simulation.

It is noteworthy to reconsider the correlation (15) and its associated constants (14) in slightly greater detail. In the case of a porous geometry with a variation parameter that is either increasing uniformly or decreasing uniformly, the upstream and downstream changes in variation parameters of each layer will have the same magnitude but opposite signs, $\Delta \beta_{i}^{+}=-\Delta \beta_{i}^{-}$. Since there is similarity in the constants of (14) $a_{4} \approx a_{6}$ and $a_{5} \approx a_{7}$, when $\Delta \beta_{i}^{+}=-\Delta \beta_{i}^{-}$, the last two terms the correlation (15) nearly cancel each other. In this way for a medium with a constant upstream change in variation parameter, the correlation may be further reduced to the three terms:

$$
\frac{K_{i}}{L_{0}^{2}} \approx a_{1}+a_{2} \beta_{i}+a_{3} \beta_{i}^{2} \quad i=1,2, \ldots, N
$$


Fig. 9 Non-uniform change in variation parameter

$\Delta \beta_{i}^{+} \neq-\Delta \beta_{i+1}^{-}$. The

comparison between the results of numerical simulation and of the full correlation of Eq. (15) of a the difference in average pressure across each layer, and $\mathbf{b}$ the pressure at each layer outlet
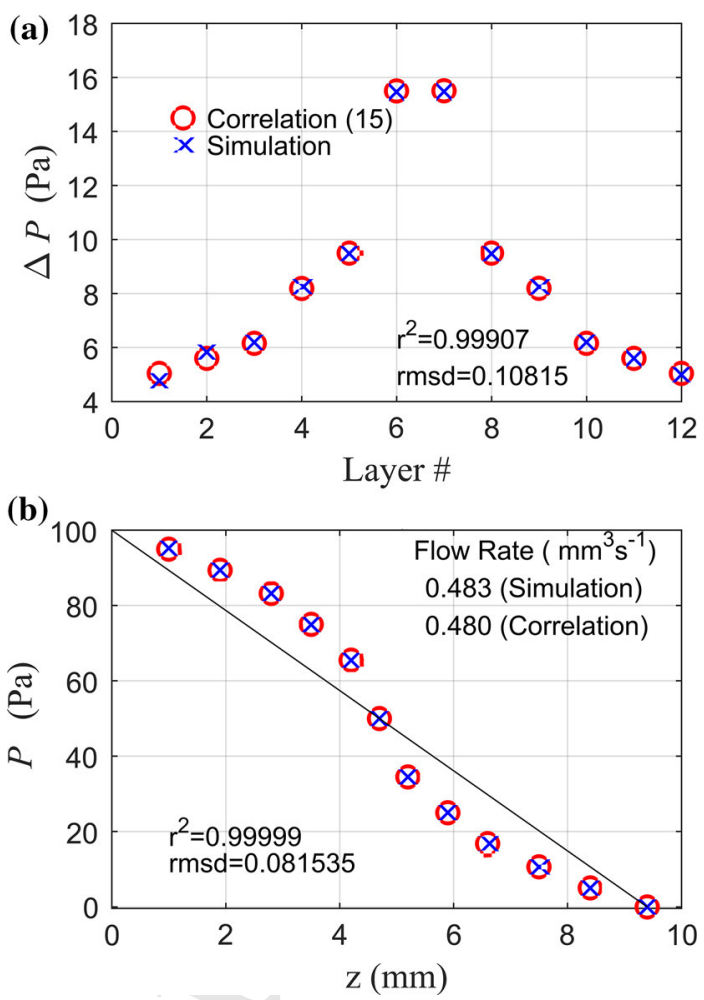

To illustrate the validity of this approximation, the permeability values resulting from correlation (18) are used with Eqs. (16) and (17) in order to predict the flow rate, pressure drop over each layer, and pressure at each layer's outlet. Comparisons to the simulation are presented Fig. 8. The reduced three term correlation of Eq. (18) results in no significant increase in error in the predictions of the local pressure drops or in the predictions of interface pressures. The error associated with prediction of the volumetric flow rate increases only slightly to $1.2 \%$. This is an important finding because the determination of the first three constants of Eq. (13) require fewer simulations compared to that required in the determination of all 7 constants. In fact, according to the preliminary results (recall Fig. 5a), the permeability dependence on variation parameter for a uniform medium is nearly perfectly quadratic in the range $0 \leq \beta \leq 0.5$. Thus, for any similar medium with a uniformly increasing or decreasing pore structure, as few as 3 simulations could be used to determine an accurate correlation to predict pressure drop across each layer and the volumetric flow rate.

A second test case considers the 12 layer structure depicted in Fig. $6 \mathrm{~b}$ that experiences non-uniform increases and decreases in characteristic pore lengths such that for each layer: $\Delta \beta_{i}^{+} \neq-\Delta \beta_{i}^{-}$. Here, the upstream and downstream differences in the variation parameter reach the maximum magnitude of 0.2 . The variation parameters are represented by the array:

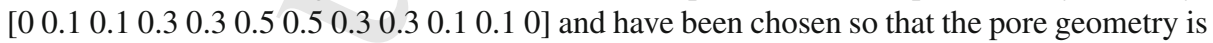
symmetric about the axial mid-plane of the medium. Comsol simulations of the flow through this geometry are conducted and the predictions of the correlation resulting from Eqs. (13)(17) are evaluated. A comparison between the two is made and presented in Fig. 9. Here, the 
Fig. 10 Non-uniform change in variation parameter $\Delta \beta_{i}^{+} \neq-\Delta \beta_{i+1}^{-}$. The comparison between Numerical Simulation and the Reduced Correlation of Eq. (18) of a the difference in average pressure across each layer, and $\mathbf{b}$ the pressure at each layer outlet
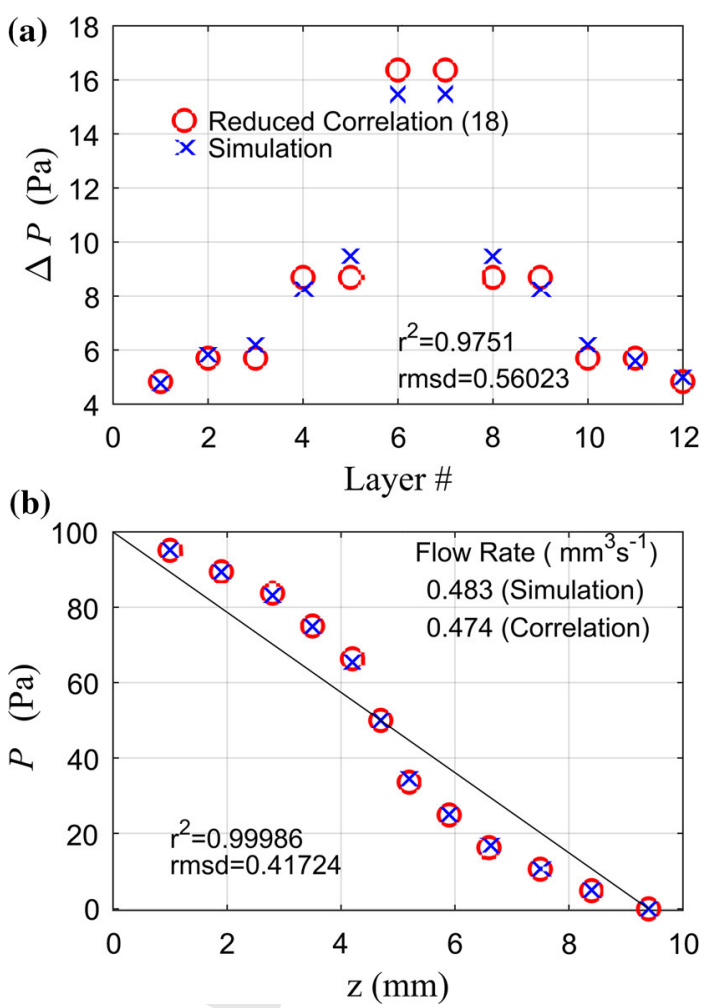

magnitude of the pressure difference across each layer increases then decreases reflecting the increasing and decreasing magnitudes of $\beta$. Again the correlation's predictions of the pressure drop across each layer and of the average pressure at each layer's outlet result in excellent agreement with those determined from the results of the full Navier-Stokes simulations. The predicted volumetric flow rate of Eq. (16) agrees to within $1 \%$ of that determined from the numerical simulation.

Following the implications of the uniformly increasing geometry of the first test case, the reduced three term correlation of Eq. (18) is implemented to determine the permeability values used in Eqs. (16) and (17). The depiction of the comparison to the simulation are presented in Fig. 10. The reduced correlation of the pressure drop across each layer results in lower agreement to the simulations (Fig. 10a). However, the reduced correlation does still provide good approximations to the average pressures at the layer interfaces (Fig. 10b). This may be explained by inspecting the pressure differences of Fig. 10a in detail. The correlation's under prediction of the pressure drop of one layer is balanced by its over prediction in the pressure drop of the adjacent layer. Since the addition of adjacent layers' pressure drops are implicit in the evaluation of the actual pressure at the layer interfaces, the errors associated with the predicted pressure drops cancel out. While still low, the root-mean-square difference between the pressures determined by the reduced three term correlation and those determined by the numerical simulation is an order of magnitude higher than that of the full correlation. The error in the predicted flow rate increases to nearly $2 \%$.

\section{黑 Springer}




\section{Conclusions}

A correlation is developed that relates local permeability to local pore geometry in a nonuniform porous medium for which the changes in local pore geometry may be characterized by a single parameter, $\beta$. Given only the information of the fluid viscosity, the matrix geometry, and total pressure drop, the correlation is able to accurately predict the volumetric flow rate and the pressure drop over each layer. When the variation parameter increases or decreases uniformly between layers, a reduced 3 term correlation (whose constants may be evaluated from only three simulations) results in only slightly less accuracy in the prediction of each layer's pressure drop and of the volume flow rate. The method of the present work is easily extended to develop correlations for variations on the current parallelepiped geometry or for pore structures of entirely different geometries. Future work could, for example, consider this method to predict pressure losses in media composed of stacks of spheroids that experience variations in the equatorial and polar radii. Future work should also consider an analysis of the thermal profile in order to develop a correlation relating the local Nusselt number to the variation parameter.

\section{Appendix}

The correlation presented in this study was evaluated from the numerical solution to the steady incompressible Continuity and Navier-Stokes Equations

$$
\begin{aligned}
& \nabla \cdot \mathbf{u}=0 \\
& \rho(\mathbf{u} \cdot \nabla) \mathbf{u}=-\nabla p+\mu \nabla^{2} \mathbf{u}
\end{aligned}
$$

The numerical solution of the axial velocity component determined from simulation is presented in Fig. 11 for the flow through a uniform six layer pore structure for which $\beta_{i}=0 i=1, \ldots, 6$. The total pressure drop is $100 \mathrm{~Pa}$, the density is $10^{3} \mathrm{~kg} \mathrm{~m}^{-3}$ and the viscosity is $0.1 \mathrm{Pas}^{-1}$.

In the simulations of the 6 layer structure, the boundary conditions at the inlet and outlet are specified pressure conditions and such uniform pressure distributions will not be found at the layer interfaces in the interior of the domain. It is the intent here to show that the local permeability of the inner layers (especially of layer 3 and of layer 4) are domain

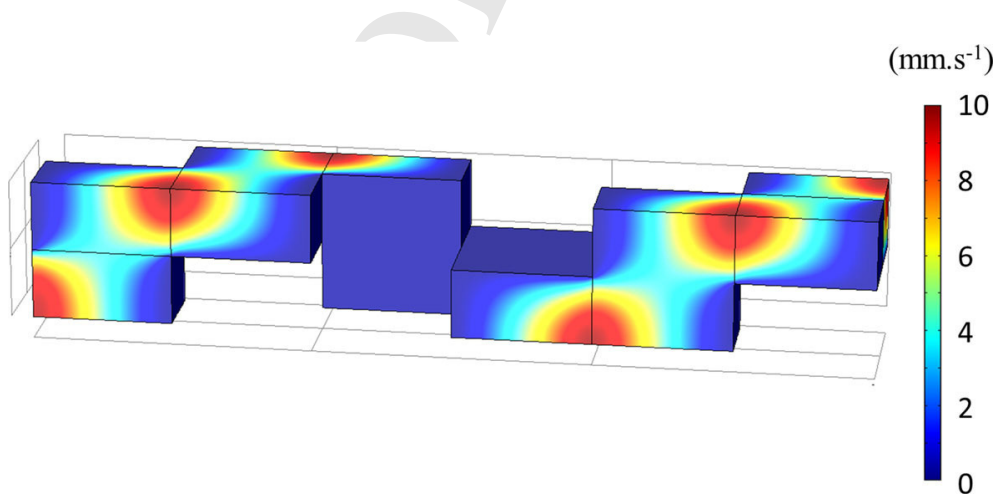

Fig. 11 Contour plot of the numerical flow velocity (in the direction of bulk flow) along the outer surface of a 6 layer structure 

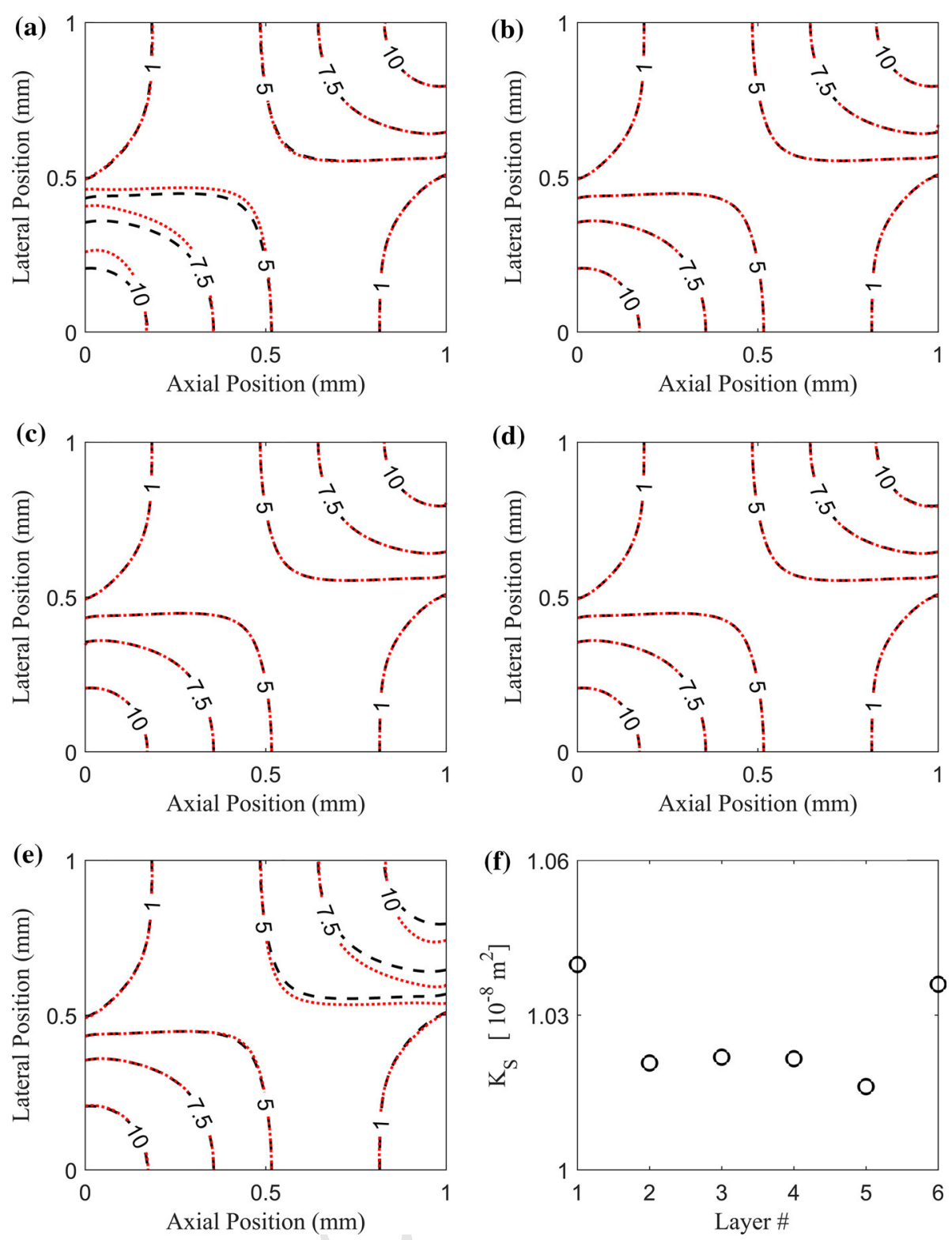

Fig. 12 Contour plots of the flow velocity $(\mathrm{mm} / \mathrm{s})$ along the outer plane of symmetry of different layers. In all panes the black dashed line corresponds to the velocity contours of Layer 2. In each panel, the red dotted line corresponds to the velocity contours of a Layer 1, b Layer 3, c Layer 4, d Layer 5, e Layer 6. In panel f the local permeability of each layer calculated from simulation data using Eq. (12)

independent. It is anticipated that at such low $R e$, any variations in the flow profile resulting from these boundary conditions will dampen out within 1-2 pore lengths. This may be explicitly demonstrated by comparing the flow profiles within the medium at selected periodic regions. Here, the $1 \mathrm{~mm} \times 1 \mathrm{~mm}$ surface corresponding a plane of symmetry of each layer is

\section{Springer}


chosen as the representative region. Profiles of the contours of the $z$ component of velocity along these planes at different layers are superimposed upon one another in Fig. 12. In each pane of Fig. 12, the black dashed contour lines correspond to the velocity of layer 2 . The red dotted line corresponds to the velocity contours of the remaining layers. The velocity profile of the inner layers are indistinguishable from one another. Only the flow fields of the outer layers (layer 1 and layer 6) are noticeably different from layer 2. This shows that at such low $R e$, the variation to the flow profile introduced by the boundary condition at the inlet of the first layer, only appears in the first layer. Similarly, the variation to the flow profile introduced by the outlet boundary condition of the last layer, only appears in the last layer. To conclude, the local permeability values calculated from the simulation results using Eq. (12) are plotted for each layer in Fig. 12f. The very small deviations in permeability values of the outer layers are not apparent in the interior layers. This helps to establish the domain independence of the calculated permeability values of layer 3 and layer 4 determined from the simulations of flow through 6 layer structures.

\section{References}

Azzam, M.I.S., Dullien, F.A.L.: Flow in tubes with periodic step changes in diameter: a numerical solution. Chem. Eng. Sci. 32(12), 1445-1455 (1977)

Balankin, A.S., Valdivia, J.-C., Marquez, J., Susarrey, O., Solorio-Avila, M.A.: Anomalous diffusion of fluid momentum and Darcy-like law for laminar flow in media with fractal porosity. Phys. Lett. A 380(35), 2767-2773 (2016)

Barrer, R.M., Petropoulos, J.H.: Diffusion in heterogeneous media: lattices of parallelepipeds in a continuous phase. Br. J. Appl. Phys. 12(12), 691-697 (1961)

Dullien, F.A.L., Azzam, M.I.S.: Effect of geometric parameters on the friction factor in periodically constricted tubes. AIChE J. 19(5), 1035-1036 (1973)

Dullien, F.A.L., Azzam, M.I.S.: Comparison of pore size as determined by mercury porosimetry and by miscible displacement experiment. Ind. Eng. Chem. Fundam. 15(2), 147-147 (1976)

Dullien, F.A.L., Elsayed, M.S., Batra, V.K.: Rate of capillary rise in porous-media with nonuniform pores. J. Colloid Interface Sci. 60(3), 497-506 (1977)

Goyeau, B., Benihaddadene, T., Gobin, D., Quintard, M.: Averaged momentum equation for flow through a nonhomogeneous porous structure. Trans. Porous Med. 28(1), 19-50 (1997)

Goyeau, B., Gobin, D., Benihaddadene, T., Gobin, D., Quintard, M.: Numerical calculation of the permeability in a dendritic mushy zone. Metal. Mater. Trans. B 30(4), 613-622 (1999)

Mathieu-Potvin, F., Gosselin, L.: Impact of non-uniform properties on governing equations for fluid flows in porous media. Transp. Porous Med. 105(2), 277-314 (2014)

Mazaheri, A.R., Zerai, B., Ahmadi, G., Kadambi, J.R., Saylor, B.Z., Oliver, M., Bromhal, G.S., Smith, D.H.: Computer simulation of flow through a lattice flow-cell model. Adv. Water Resour. 28(12), 1267-1279 (2005)

Munro, B., Becker, S., Uth, M.F., Preusser, N., Herwig, H.: Fabrication and characterization of deformable porous matrices with controlled pore characteristics. Transp. Porous Med. 107(1), 79-94 (2015)

Nield, D.A., Bejan, A.: Convection in Porous Media. Springer, New York (2013)

Tehlar, D., Flückiger, R., Wokaun, A., Büchi, F.N.: Investigation of channel-to-channel cross convection in serpentine flow fields. Fuel Cells 10(6), 1040-1049 (2010)

Vafai, K.: Convective flow and heat transfer in variable-porosity media. J. Fluid Mech. 147(1), 233-259 (1984) 
Journal: 11242

Article: 998

\section{焦 Springer}

the language of science

\section{Author Query Form}

\section{Please ensure you fill out your response to the queries raised below and return this form along with your corrections}

\section{Dear Author}

During the process of typesetting your article, the following queries have arisen. Please check your typeset proof carefully against the queries listed below and mark the necessary changes either directly on the proof/online grid or in the 'Author's response' area provided below

\begin{tabular}{|c|l|l|}
\hline Query & \multicolumn{1}{|c|}{ Details required } & Author's response \\
\hline 1. & $\begin{array}{l}\text { Edit made in article title kindly check and } \\
\text { confirm. }\end{array}$ & \\
\hline 2. & $\begin{array}{l}\text { Reference Dullien (1992) was mentioned } \\
\text { in the manuscript; however, this was } \\
\text { not included in the reference list. As a } \\
\text { rule, all mentioned references should be } \\
\text { present in the reference list. Please pro- } \\
\text { vide the reference details to be inserted } \\
\text { in the reference list. }\end{array}$ & \\
\hline
\end{tabular}

